\title{
New Avenues for the Development of Therapeutics
}

National Cancer Institute

\section{Source}

National Cancer Institute. New Avenues for the Development of Therapeutics. NCI

Thesaurus. Code C15883.

NIH Emphasis Area -- The result of efforts in chemistry, structural biology, genetics, and cellular and molecular biology are providing new means to design therapies for a variety of diseases. Efforts to discover and develop drugs to combat cancer and drug and alcohol addiction and methods of bioeng ineering to repair tissues are ripe for discovery. 